\title{
UK neonatal intensive care services in 1996
}

Janet Tucker, William Tarnow-Mordi, Craig Gould, Gareth Parry, Neil Marlow, on behalf of the UK Neonatal Staffing Study Collaborative Group

\begin{abstract}
A census of activity and staff levels in $\mathbf{1 9 9 6}$ was conducted in UK neonatal units and achieved a $100 \%$ response from 246 units. Among the 186 neonatal intensive care units, the median (interquartile range) number of total cots was 18(14-22); level 1 intensive care cots $4(2-6)$; total admissions 318(262-405); very low birthweight admissions 40(28-68); and the number ventilated or given CPAP by endotracheal tube $52(32-83)$. Forty six $(25 \%)$ intensive care units lacked the recommended minimum of one consultant with prime responsibility for neonatal medicine. As a conservative estimate $79 \%$ of intensive care units had a lower nursing provision than that recommended in previously published guidelines. There was substantial variation in activity and staffing levels among units.

(Arch Dis Child Fetal Neonatal Ed 1999;80:F233-F234)
\end{abstract}

Keywords: staff numbers; intensive care units; risk adjusted outcomes

On behalf of the British Association of Perinatal Medicine (BAPM), Milligan ${ }^{1}$ surveyed neonatal intensive care provision in the UK for 1992-93; the response rate reached $84 \%$. He reported increased numbers of intensive care level 1 cots and increased intensive care activity compared with 1989. However, contrary to contemporary policy initiatives, most of the neonatal intensive care activity was delivered in small or medium sized units.

In 1996 the $\mathrm{BAPM}^{2}$ offered guidance on organisation and standards, and recommended that units offering neonatal intensive care should have total level 1 and level 2 activity of over 500 intensive care days per year. However, BAPM also emphasised the need to obtain evidence that implementation of the standards, including the still contentious issue of size and throughput of units, would improve outcomes.
The UK Neonatal Staffing Study aims to address this issue and we report here the results of the first census phase.

We achieved responses from every UK neonatal unit, describing patient volume, activity levels and throughput, nurse and medical staff numbers. These results gave a complete sampling frame for a stratified random sample of UK neonatal intensive care units for the second prospective phase. That second phase will consider the relation between the directly alterable characteristics of size, staffing levels and workload and risk adjusted mortality and morbidity.

\section{Methods and results}

Of the 250 UK hospitals surveyed in 1997, three had closed and two had merged. All remaining 246 units returned questionnaires using their most recent annual data available (98\% for 1996, 2\% for 1995). One hundred and eighty six $(76 \%)$ were categorised as neonatal intensive care units because they reported providing sustained neonatal intensive care as well as special care. The remaining $60(24 \%)$ were special care baby units which provided only temporary intensive care before transfer to a neonatal intensive care unit.

\section{ACTIVITY}

The reported cot establishment and descriptions of unit activity for 186 neonatal intensive care units are summarised in table 1. Definitions used to measure ventilation activity varied between units, both for the total number of infants ventilated or given continuous positive airway pressure (CPAP) and for the total counts of ventilated days. A few units counted ventilation activity only as that delivered by endotracheal tube, whereas most included other respiratory support, such as that delivered by nasal prongs or face mask. Thus the most complete, comparable, and robust proxy measure available of neonatal intensive care activity was total annual admissions of very low
Mealth, Ninewells Medical School,

University of Dundee,

Dundee DD1 9SY

J Tucker

W Tarnow-Mordi

C Gould

Medical Care Research Unit, University of Sheffield

G Parry

Department of Child Health, University Hospital, Queen's

Medical Centre,

Nottingham

$\mathrm{N}$ Marlow

Correspondence to:

Dr Janet Tucker.

Accepted 20 November 1998
Table 1 Reported cot establishment and activity levels in UK neonatal intensive care units

\begin{tabular}{|c|c|c|c|}
\hline Establishment and activity variables (reported per anпum) & $\begin{array}{l}\text { Median (interquartile } \\
\text { range) }\end{array}$ & $\begin{array}{l}\text { Minimum-maximum } \\
\text { range }\end{array}$ & $\begin{array}{l}\text { NICU total } 186 \\
(\% \text { item response) }\end{array}$ \\
\hline Total number of admissions & $318(262-405)$ & $48-1020$ & $186(100 \%)$ \\
\hline Total number of cots in NICU & $18(14-22)$ & $4-55$ & $186(100 \%)$ \\
\hline Total number of IC level 1 cots & $4(2-6)$ & $0 \star-16$ & $186(100 \%)$ \\
\hline Total number of infants ventilated or given CPAP & & & Total $174(94 \%)$ \\
\hline Counts only infants supported by endotracheal tube & $52(32-83)$ & $10-269$ & $33(18 \%)$ \\
\hline $\begin{array}{l}\text { Counts infants supported by endotraceal tube, face mask or } \\
\text { nasal prongs }\end{array}$ & $66(40-113)$ & $12-310$ & $141(76 \%)$ \\
\hline Total number of ventilator days & & & Total $150(80 \%)$ \\
\hline Counts only for support by endotracheal tube & $281(139-817)$ & $19-2688$ & $34(18 \%)$ \\
\hline $\begin{array}{l}\text { Counts for support by endotracheal tube, face mask and nasal } \\
\text { prongs }\end{array}$ & $451(205-968)$ & $13-3324$ & $116(62 \%)$ \\
\hline Total number of VLBW infants admitted & $40(28-68)$ & $2-227$ & $182(95 \%)$ \\
\hline
\end{tabular}

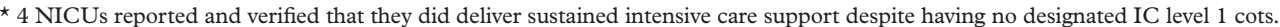
$\mathrm{CPAP}=$ continuous positive airway pressure; VLBW = very low birthweight $(<1500 \mathrm{~g})$. 


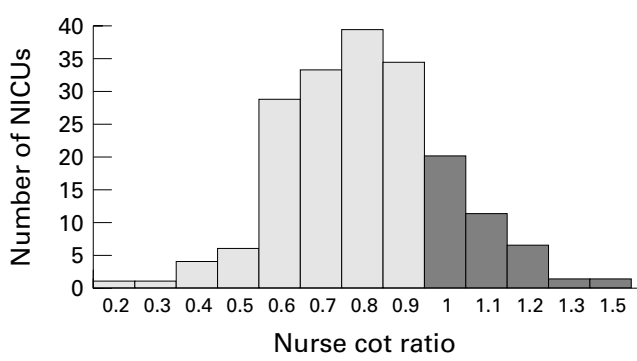

Figure 1 Nurse:cot ratios in all UK neonatal intensive care units (actual/recommended numbers of WTE nurses).

birthweight (VLBW) babies. With the development and adoption of the BAPM neonatal dataset, for annual reporting of data for neonatal intensive care units, ${ }^{3}$ more reliable data will be routinely available for future comparisons of unit based activity and staffing.

STAFFING

Forty six $(25 \%)$ neonatal intensive care units lacked the recommended minimum of one consultant ${ }^{1}$ with prime responsibility for neonatal medicine (measured as $>50 \%$ of clinical sessions dedicated to neonatal care). The trained and qualified nurse staffing establishment proposals of $1992^{4}$ were 5.5 whole time equivalent (WTE) nurses per level 1 intensive care cot, 3.5 per level 2 intensive care cot, and one per special care cot. We used more conservative assumptions (fig 1) for two reasons. Firstly, this census did not distinguish between level 2 intensive care cots and special care cots, and secondly it did not distinguish between trained nurses, those with additional qualifications in neonatal specialty, nursery nurses and auxiliaries. A ratio of 1.0 indicates reported nursing establishment provision equivalent to the calculated requirement: $79 \%$ $(147 / 186)$ of neonatal intensive care units had ratios <1.0, varying from 0.3-1.5 (median 0.84, IQR 0.73-0.98) (fig 1).

Nurse:cot ratios (fig 1) were calculated using the following formula: WTE/(5.5 $\times$ ICL1+SC), where WTE is observed total whole time equivalent nurses in the establishment, including trained, qualified nurses and auxiliary or nursery nurses; ICL1 = total level 1 intensive care cots; and SC $=$ total number of other cots, such as level 2 intensive care cots and special care cots. Seventy nine per cent of neonatal intensive care units had a nurse:cot ratio $<1$, indicating lower provision than that required by our conservative interpretation of the 1992 guidelines. The calculation underestimates the deficit between actual and recommended numbers of nurses, as it equates trained, qualified, and untrained nurses, and level 2 intensive care with special care cots.

\section{Comment}

These results show continuing and substantial variation in neonatal intensive care unit activity and staff numbers. ${ }^{1}$ Despite conservative assumptions in our calculation, we noted divergence between actual and calculated nurse requirement. Similar wide variation has been described by Redshaw and colleagues. ${ }^{5}$ The reasons for the variation in levels of activity probably include different admission criteria and inherent differences in case mix. The reasons for divergence in staffing provision could include varying definitions of cot types and categories of care, ${ }^{6}$ resource constraints, problems in recruitment or retention of staff, and doubts about the validity of the staffing guidelines. $^{4}$

Whether these observed variations in organisational characteristics are important determinants of outcome remains unknown. It has been suggested that staff may get more experience if activity and specialisation are high, ${ }^{1}$ but outcomes may deteriorate if staff are overworked. In the second phase of this study risk adjusted outcomes will be assessed prospectively for over 6000 infants from a random sample of 54 neonatal intensive care units, stratified by these census measures of activity (volume of VLBW babies), and medical and nurse staffing numbers. Consistent measures of workload and staffing ${ }^{6}$ will also be observed and recorded twice daily. The UK Neonatal Staffing Study may provide more reliable evidence to inform policy than has been available before.

Website addresses for information and protocol of the UK Neonatal Staffing Study: child-health.dundee.ac.uk/research/ UKneonatal-staffing thelancet.com

The UK Neonatal Staffing study is funded by the NHS R\&D Executive Mother and Child Health Programme and endorsed by the British Association of Perinatal Medicine, the Neonatal Nurses Association (UK) and Scottish Neonatal Nurses Group. We thank all UK neonatal units for their support. The views We thank all behalf of the UK Neonatal Staffing Study Group.

1 Milligan DWA. Neonatal intensive care provision in the United Kingdom 1992-3. Arch Dis Child Fetal United Kingdom

2 British Association of Perinatal Medicine. Standards for Hospitals Providing Neonatal Intensive Care. London: BAPM, 1996.

3 British Association of Perinatal Medicine. The BAPM neonatal dataset for the annual reporting of data by neonatal intensive care units. Report of a Working Party of the British Association of Perinatal Medicine. London: BAPM, 1997.

4 Report of working group of the British Association of Perinatal Medicine and Neonatal Nurses Association on categories of babies requiring neonatal care. Arch Dis Child 1992;67:868-9.

5 Redshaw ME, Harris A, Ingram JC. Delivering neonatal care. The neonatal unit as a working environment: a survey of neoThe neonatal unit as a working environment:

6 The ECSURF (Economic Evaluation of Surfactant) Collaborative Study Group. Limited comparability of classifications of levels of neonatal care in UK units. Arch Dis Child 1998; 78:F179-F84. 